\title{
Near-infrared analysis of nanofibrillated cellulose aerogel manufacturing
}

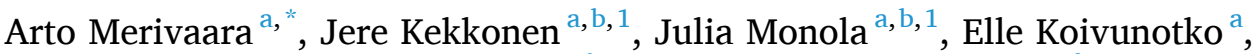 \\ Marko Savolainen ${ }^{c}$, Tuomo Silvast ${ }^{\mathrm{d}}$, Kirsi Svedström ${ }^{\mathrm{e}}$, Ana Diaz ${ }^{\mathrm{f}}$, Mirko Holler ${ }^{\mathrm{f}}$, \\ Ossi Korhonen $^{\mathrm{b}}$, Marjo Yliperttula ${ }^{\mathrm{a}, *}$, Sami Valkonen ${ }^{\mathrm{a}, \mathrm{b}}$ \\ ${ }^{a}$ Division of Pharmaceutical Biosciences, Faculty of Pharmacy, University of Helsinki, P.O. Box 56, 00014 Helsinki, Finland \\ ${ }^{\mathrm{b}}$ School of Pharmacy, University of Eastern Finland, 70210 Kuopio, Finland \\ c Optical Measurements, VTT Technical Research Centre of Finland, Finland \\ ${ }^{\mathrm{d}}$ SIB Labs, Faculty of Science and Forestry, University of Eastern Finland, Kuopio, Finland \\ ${ }^{\mathrm{e}}$ Department of Physics, University of Helsinki, P.O. Box 64, 00014 Helsinki, Finland \\ ${ }^{\mathrm{f}}$ Paul Scherrer Institute, Forschungsstrasse 111, 5232 Villigen PSI, Switzerland
}

\section{A R T I C L E I N F O}

\section{Keywords:}

Freeze-drying

Near-infrared spectroscopy

Aerogel

Biomaterials

Nanofibrillated cellulose

Biophotonics

\begin{abstract}
A B S T R A C T
Biomaterial aerogel fabrication by freeze-drying must be further improved to reduce the costs of lengthy freezedrying cycles and to avoid the formation of spongy cryogels and collapse of the aerogel structures. Residual water content is a critical quality attribute of the freeze-dried product, which can be monitored in-line with nearinfrared (NIR) spectroscopy. Predictive models of NIR have not been previously applied for biomaterials and the models were mostly focused on the prediction of only one formulation at a time. We recorded NIR spectra of different nanofibrillated cellulose (NFC) hydrogel formulations during the secondary drying and set up a partial least square regression model to predict their residual water contents. The model can be generalized to measure residual water of formulations with different NFC concentrations and the excipients, and the NFC fiber concentrations and excipients can be separated with the principal component analysis. Our results provide valuable information about the freeze-drying of biomaterials and aerogel fabrication, and how NIR spectroscopy can be utilized in the optimization of residual water content.
\end{abstract}

\section{Introduction}

Novel drug delivery systems and treatments are ever-increasingly needed. Biomaterials, such as hydrogels or extracellular vesicles, offer versatile solutions for drug delivery, drug discovery, or tissue engineering (Cabeza et al., 2020; Cascone and Lamberti, 2020). Especially natural biomaterials are appreciated for biomedical applications due to their biocompatibility, renewability, and biodegradability (George et al., 2019). One approach to utilize natural biomaterials for drug delivery or tissue engineering is to fabricate them as aerogels (GarcíaGonzález et al., 2021; Veres et al., 2015). In general, aerogels are solid lightweight materials with extremely low density in between 0.001 and $0.2 \mathrm{~g} / \mathrm{cm}^{3}$, with over $90 \%$ porosity with the most prevalent pore diameter of $2-50 \mathrm{~nm}$ and with tunable mechanical and chemical properties (Zaman et al., 2020). Concerning the drug delivery and biomedical approaches, aerogels can be utilized for example for controlled drug release, to provide scaffolds for cell colonization, or wound healing (Blasi-Romero et al., 2021; Grenier et al., 2019; Nagy et al., 2019).

Aerogels can be fabricated from natural biomaterials, such as alginate (Athamneh et al., 2019), hyaluronic acid (Najberg et al., 2020), or nanofibrillated cellulose (NFC) (Jin et al., 2004), by solvent exchange drying, bioprinting, thermally induced phase separation, and freezedrying (Brougham et al., 2017; Chen et al., 2019; García-González et al., 2021; Jin et al., 2004; Parisi et al., 2021). In this study, we focus on the freeze-drying of NFC hydrogel. Freeze-drying in turn is a widely used desiccation method in the pharmaceutical and biomedical industry to preserve heat-sensitive products, such as protein drugs and vaccines (Franks and Auffret, 2008). The freeze-drying cycle begins with a freezing step, during which the specimen is frozen below the glass transition temperature of the maximally freeze concentrated sample ( $\mathrm{Tg}^{\prime}$ ). Then, the ice is sublimated during the primary drying, and lastly, in the secondary drying step, the unfrozen water is desorbed. When

\footnotetext{
* Corresponding authors at: Viikinkaari 5E, P.O. Box 56, 00014, University of Helsinki, Helsinki, Finland.

E-mail addresses: arto.merivaara@helsinki.fi (A. Merivaara), marjo.yliperttula@helsinki.fi (M. Yliperttula).

1 Shared second author.
} 
fabricating aerogels by freeze-drying, the end product should be a dry highly porous aerogel with firm mechanical properties. The freezing step has a major impact on the porosity of the aerogel, and to control the porosity different approaches, such as directional freezing, can be used (Qin et al., 2021). However, isotropic freezing is the most prevalent method of freezing and highly porous aerogels with regular structure can be obtained also with this approach.

To obtain an aerogel product of high quality by freeze-drying the critical process parameters, such as freezing rate and shelf temperature, and critical quality attributes, such as residual water content and porosity, must be controlled as we have recently reviewed (Merivaara et al., 2021). Water acts as a plasticizer in the freeze-dried aerogel which can result in collapsing (macro or micro collapse) of the aerogel structures. In addition, most chemical degradation reactions and microbiological contaminations require water. Furthermore, water plays a special role with hydrogels. In general, the amount of unfrozen water in the hydrogels can be high due to the nature of tightly bound unfrozen water present in the hydrogels (Gun'ko et al., 2017). This leads to timeconsuming and potentially unpredictable freeze-drying cycles when fabricating aerogels. Especially the role of secondary drying can be considered important because if the freeze-drying cycle is stopped prematurely, in the worst-case during the primary drying before all ice is sublimated, the samples would melt resulting in spongy cryogels, and no proper aerogel structure would be obtained (García-González et al., 2021). Contrary, and especially if the samples dry heterogeneously, to avoid losing the samples due to high residual water contents the freezedrying cycle must be continued "just-in-case", which causes extra expenses. The second critical quality attribute of the aerogels, porosity, facilitates for example the mass transfer i.e., how fluids (diffusion of drugs) or considering tissue engineering, cells, move in and out of the aerogel. Despite the importance of characterization of the critical quality attributes at the end of the process with for example Karl Fischer titrator for residual water content and scanning electron microscope for porosity, the utilization of process analytical technology (PAT) to monitor the freeze-drying process in-line, would be of utmost importance.

Vibrational spectroscopy offers potential methods, mainly Raman and near-infrared (NIR) spectroscopy, to be implemented as process analytical technology. Raman and NIR spectroscopies are both noninvasive non-destructive fast label-free methods suitable for at-line, on-line or in-line analysis, and they can be considered complementary methods in freeze-drying: NIR is sensitive to ice and water, while ice and water produce only poor Raman scatters. With a Raman spectroscope information about the physical state of the sample and interactions occurring can be obtained. Besides freeze-drying, both NIR and Raman spectroscopies have been earlier applied also in pharmaceutical applications as PAT for example in blending, granulation, drying, tablet manufacturing, pelletization, and extrusion of solid forms (de Beer et al., 2011).

NIR spectroscopy has been applied already in 1989 by Kamat et al. in freeze-drying to determine the residual water content of freeze-dried sucrose solutions (Kamat et al., 1989). Recently, NIR spectroscopy has been used to monitor formulation changes in a multicomponent drug formulation during freeze-drying (Rosas et al., 2014), to predict residual water content of freeze-dried monoclonal antibody formulations (Clavaud et al., 2020), and to monitor the freeze-drying of ibuprofen for injection formulations (Preskar et al., 2021). We have previously applied a multipoint NIR system to monitor in-line the residual water contents of sucrose-water solutions during freeze-drying (Kauppinen et al., 2013). NIR spectroscopy was applied to monitor in-line residual water content of sucrose formulations and partial least square (PLS) models were created for independent formulations. However, no general model of prediction for varying sucrose concentration was developed. To the best of our knowledge, no other study has applied NIR spectroscopy to monitor freeze-drying of biomaterials as a predictive method for aerogel fabrication.
In the future, more sophisticated tools to freeze-dry and to monitor freeze-drying of biomaterials are needed as we discuss in the review article by Merivaara et al. (2021) (Merivaara et al., 2021). To answer the need for improved methods, PAT must be applied to the process. Regarding the freeze-drying of biomaterials, our recent results indicate that depending on excipient and biomaterial concentrations, we obtain end products with different residual water contents and porosity (Koivunotko et al., 2021). In that study, we explained how excipients prevent aggregation of NFC hydrogel during freeze-drying facilitating its subsequent reconstitution. In addition, we have previously freeze-dried modified anionic nanofibrillated cellulose hydrogel and showed that the drug release profiles remained unaltered after freeze-drying and reconstitution (Paukkonen et al., 2017).

In this study, we showed the suitability of NIR spectroscopy for monitoring of manufacturing of biomaterial aerogels by freeze-drying and predicted their residual water content during secondary drying with PLS models. This improves the process by allowing to shorten the freeze-drying cycle and by potentially providing valuable insight into the mechanism of action of the excipients. We hypothesized that, first, the amount of residual water of the biomaterial formulation can be predicted with NIR spectroscopy, and second, the model can be generalized to varying NFC fiber concentrations.

\section{Materials and methods}

Two widely used excipients trehalose (TRE) and sucrose (SUC) (Sigma-Aldrich, Germany) (Fig. 1), were chosen for this study based on our previous results (Koivunotko et al., 2021). 1.5\% (m/V) nanofibrillated cellulose (NFC) hydrogel (GrowDex ${ }^{\circledR}$ ) was obtained from UPM-Kymmene, Finland. NFC hydrogel and excipients were mixed by syringe mixing as described by Paukkonen et al. (2017). Formulations studied in this study were $1.5 \%$ NFC with $300 \mathrm{mM}$ sucrose, $1.5 \%$ NFC with $300 \mathrm{mM}$ trehalose, $0.8 \%$ NFC with $300 \mathrm{mM}$ sucrose, and $0.8 \%$ NFC with $300 \mathrm{mM}$ trehalose. Control samples were $1.5 \% \mathrm{NFC}, 0.8 \%$ NFC, $300 \mathrm{mM}$ sucrose water (Milli-Q ${ }^{\circledR}$ ) solution, and $300 \mathrm{mM}$ trehalose water (Milli-Q®) solution.

\subsection{Freeze-drying process and sampling during the freeze-drying cycle}

Prior to freeze-drying, the glass transition temperature of the maximally freeze concentrated sample ( $\mathrm{Tg}$ ') of the studied formulations was determined with differential scanning calorimetry (DSC) (Discovery 2500, TA instruments, Germany) to ensure correct primary drying temperature and to prevent collapsing of the samples as reported in Koivunotko et al. (2021).

Samples were freeze-dried with LyoStar II (SP scientific Inc., USA) in $6 \mathrm{ml}$ ISO Clear Type I Tubular Glass Vial 2 -freeze-drying vials (Adelphi, UK). All samples and vials were weighed before freeze-drying. Samples were first frozen by decreasing the temperature by $1{ }^{\circ} \mathrm{C} / \mathrm{min}$ until $-47^{\circ} \mathrm{C}$ and keeping the temperature at $-47^{\circ} \mathrm{C}$ for $2 \mathrm{~h}$ (freezing step). After the freezing step, the pressure was decreased to 50 mTorr and the temperature was increased to $-42^{\circ} \mathrm{C}$ by $1{ }^{\circ} \mathrm{C} / \mathrm{min}$ (primary drying). The endpoint of the primary drying was determined by convergence of the pressure values of Pirani and capacitance manometer vacuum sensors. During the secondary drying, the temperature was increased by $1{ }^{\circ} \mathrm{C} /$ min from $-42{ }^{\circ} \mathrm{C}$ to $20^{\circ} \mathrm{C}$. The samples were collected during the secondary drying at chosen temperature points $(-42,-32,-22,-17-12$, $-7-2,+3,+8,+13$ and $+20{ }^{\circ} \mathrm{C}$ ) through a sample extractor door (SP Scientific Inc., USA) as described by Kauppinen et al. (2013), and subsequently measured with NIR spectrometer and Karl Fischer titrator (899 Coulometer, Metrohm, Switzerland). The temperature and pressure were allowed to stabilize for $45 \mathrm{~min}$ after each sample extraction, after which the temperature increasing was continued. Freeze-drying cycles are shown in the Supplementary information Fig. S1. All samples were visually inspected to observe any cake defects such as collapse, shrinkage, or cracking. 
<smiles>OC[C@H]1O[C@H]2O[C@@H]([C@H](O)[C@H](O)[C@H]2O)[C@H](O)[C@H]1O</smiles>

Trehalose (a-D-glucopyranosyl-a-D-glucopyranoside)

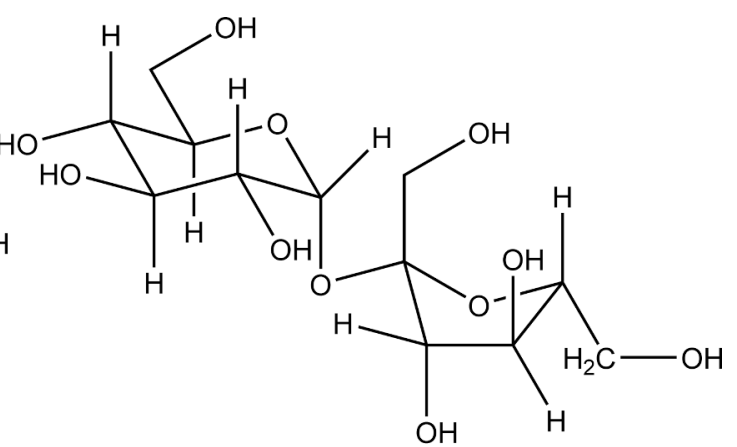

Sucrose ( $\beta$-D-fructofuranosyl-a-D-glucopyranoside)

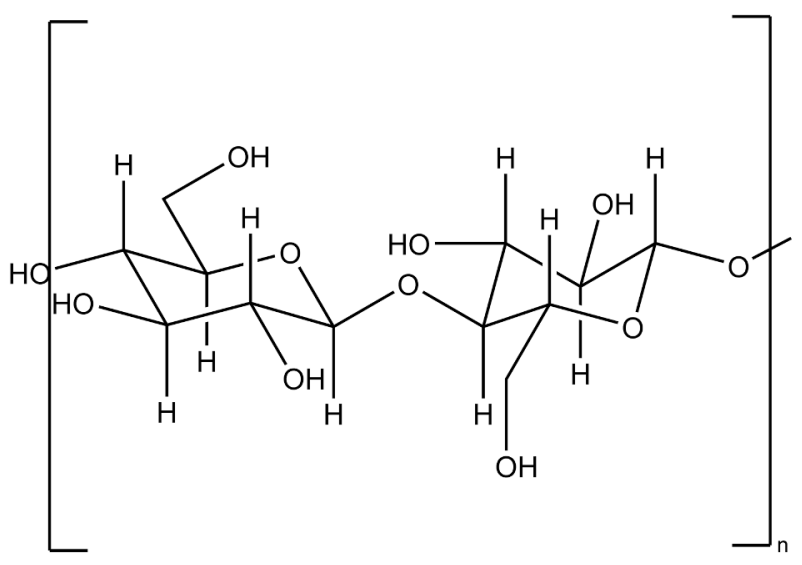

Cellulose

Fig. 1. Molecular structure of trehalose, sucrose, and cellulose in chair conformations with hydrogens.

\subsection{Characterization of NFC aerogel formulations}

The morphology of the NFC aerogels with different excipients was evaluated with a scanning electron microscope (SEM) (Quanta FEG250, FEI Company, USA). The samples were carefully shredded with tweezers to reveal the inner structure of the aerogel, placed on the two-sided carbon tape with silver paint, and sputtered with platinum for $25 \mathrm{~s}$ with an Agar sputter instrument (Agar Scientific Ltd., U.K.). Samples were imaged in high vacuum with $5-6 \mathrm{kV}$ and 3.5 spot size.

$\mathrm{X}$-ray micro computed tomography ( $\mu$-CT) imaging was performed with Nikon XT H 225 (Nikon Metrology NV) microCT X-ray tomography device. Prior to imaging, the sample was kept at room temperature for 3 $\mathrm{h}$ in order to stabilize the material. The sample was placed on a sample holder and imaged with $90 \mathrm{kV}$ (peak) voltage, image pixel size of $4 \mu \mathrm{m}$, and with 4476 projections. 16-bit .tiff images were converted to 8-bit . bmp images, binarized, and analyzed in 3D with CT Analyzer (Bruker Belgium N.V.).

The morphology and electron density of $1.5 \% \mathrm{NFC}+300 \mathrm{mM}$ SUC, $0.8 \% \mathrm{NFC}+300 \mathrm{mM} \mathrm{SUC}$, and $1.5 \%$ NFC aerogels were studied in more detail with ptychographic X-ray computed tomography (PXCT) utilizing the OMNY instrument (Holler et al., 2018) at the cSAXS beamline at the Swiss Light Source, Paul Scherrer Institut (PSI), Switzerland. The experimental setup is described in detail in Koivunotko et al. (2021), and the measurement and reconstruction parameters for the $0.8 \%$ NFC + $300 \mathrm{mM}$ SUC and $1.5 \% \mathrm{NCF}$ aerogel samples were also reported in that work. The $1.5 \%$ NFC $+300 \mathrm{mM}$ SUC aerogel was placed at $2.4 \mathrm{~mm}$ downstream the focus, where the beam was about $8 \mu \mathrm{m}$ in size, and the sample was scanned following the positions of a Fermat spiral with an average step size of $2 \mu \mathrm{m}$. For this specimen, standard far-field ptychographic reconstructions were using the PtychoShelves reconstruction package (Wakonig et al., 2020). For the reconstructions, $480 \times 480$ pixels of the detector were used, resulting in a pixel size of $40.0 \mathrm{~nm}$ in the reconstructed images. The measurement parameters, dose, and resolution for the different samples are shown in more detail in the Supplementary information Table S1. PXCT provides the 3D electron density distribution within a specimen with quantitative absolute contrast (Diaz et al., 2012). The results and quantitative electron densities, obtained from tomographic reconstructions, were visualized with VolumeViewer of Fiji.

\subsection{Residual water reference measurements with Karl Fischer titrator}

Residual water contents of the freeze-dried samples were analyzed with a coulometric Karl Fischer titrator (899 Coulometer, Metrohm, Switzerland). Hydranal (Coulomat AG, Fluca) was used as a solvent in the titrator. Before the measurements, background residual water from the methanol was measured. For the samples, first, the dry samples with vials were weighted. Then, the samples were reconstituted with $1 \mathrm{ml}$ of anhydrous methanol (Sigma-Aldrich, Germany), after which $700 \mu \mathrm{l}$ of sample dissolved in methanol was added to the titrator. Residual water contents were calculated as mass percentages (water content/dry weight).

\subsection{Near-infrared spectroscopy}

A similar custom-made NIR-spectrometer setup was used as in 
Kauppinen et al. (2013). The NIR-spectrometer consisted of a shortwavelength infrared (SWIR) hyperspectral camera (wavelength region $970 \mathrm{~nm}-2500 \mathrm{~nm}$ ) (Specim Oy, Finland) connected to a multichannel fiber-optic input module (VTT, Finland), a multichannel fiber-optic light source containing a $65 \mathrm{~W}$ halogen lamp (VTT, Finland) and a fiber-optic noncontact diffusion reflectance probe head (VTT, Finland). The illumination spot of each probe ( $\varnothing 2 \mathrm{~mm}$ ) was adjusted to measure through the side of the vial as close to the bottom of the sample as possible. The samples were collected during secondary drying as described above. The samples for the partial least square (PLS) training set and crossvalidation set were freeze-dried in the same freeze-drying cycle. NIR spectra of three parallel samples at each temperature point were recorded for $10 \mathrm{~s}$ with a $100 \mathrm{~Hz}$ frame rate.

\subsection{Data analysis}

A region of 1376-2169 $\mathrm{nm}$ of the NIR spectra was used for principal component analysis (PCA) and partial least square (PLS) models. Data processing and analysis were performed with the Unscrambler ${ }^{\circledR} \mathrm{X}$ -software (CAMO Software, Norway). Before analysis, the spectra were first smoothed with Savitzky-Golay smoothing (Polynomial Order: 1, Smoothing Points: 11, Left Points: 5, Right Points: 5). After smoothing, the spectra were subjected to 1st order Savitzky-Golay derivative transform (Derivative Order: 1, Polynomial Order: 2, Smoothing Points: 7, Left Points: 3, Right Points: 3). Lastly, the data were corrected with standard normal variate (SNV) correction.

After the preprocessing, the PCA was performed to observe any outliers, to follow the decrease of water during the secondary drying and to separate different formulations from each other, and to observe major regions of interest which caused variation to the dataset. The scores plots were compared and loading plots were interpreted with the obtained spectra. PCA was performed for all samples and the spectral region of $1376-2169 \mathrm{~nm}$.

For PLS regression analysis, first, the spectra were listed according to the sampling temperature points ( 3 parallel samples each temperature point, 11 temperature points, 4 formulations) with corresponding residual water contents. Then, every other spectrum was selected to the training set, which was used to build the PLS regression model, and the remaining half of the spectra were used for the cross-validation set. Before the validation with the cross-validation set, the model was evaluated with leave-one-out cross-validation. The accuracy of the PLS model was evaluated with a root-mean-square error of cross-validation (RMSECV) and relative standard error of cross-validation (RSECV) when the cross-validation set was used, and with a root-mean-square error of prediction (RMSEP) and relative standard error of prediction (RSEP), when external validation was used. All data were plotted with OriginPro 2020 software (OriginLab, USA).

\section{Results}

\subsection{Near-infrared spectra of NFC hydrogel excipient formulations reveal residual water contents}

NIR spectra of all NFC hydrogel formulations were recorded during the secondary drying at chosen 11 temperature points to analyze the decreasing residual water content and any differences between the drying of different NFC hydrogel formulations. The smoothed and SNV corrected first derivative spectra were inspected. All formulations show a clear decrease in absorbance between temperature points at the region of 1830-1940 nm that corresponds to the absorbance band of water (Kamat et al., 1989) (Figs. 2-5). At wavelength $1940 \mathrm{~nm}$ an isosbestic point can be observed which is also the $\mathrm{x}$-axis intercept point i.e. the local maximum of non-derivative spectra does not shift during secondary drying. The residual water contents of the different formulations during the secondary drying are shown in chapter 3.2.

Water shows another strong absorbance region at $1400-1450 \mathrm{~nm}$ (Weyer and Lo, 2006). In addition, the 1st overtone of OH-stretching is observed at that region. The derivative spectra intercept the $\mathrm{x}$-axis at 1450-1460 nm, which means again that stationary point (local maximum) of nonderivative spectra is observed at that wavelength. Minor differences were observed in that region between temperature points and formulations. The region of the 1st overtone of $\mathrm{CH}$-group can be observed at 1700-1800 nm (Weyer and Lo, 2006).

The physical properties of the material will change during the freezedrying process for example from crystalline to amorphous. This can be observed as the shift from lower energy (higher wavelength) to higher

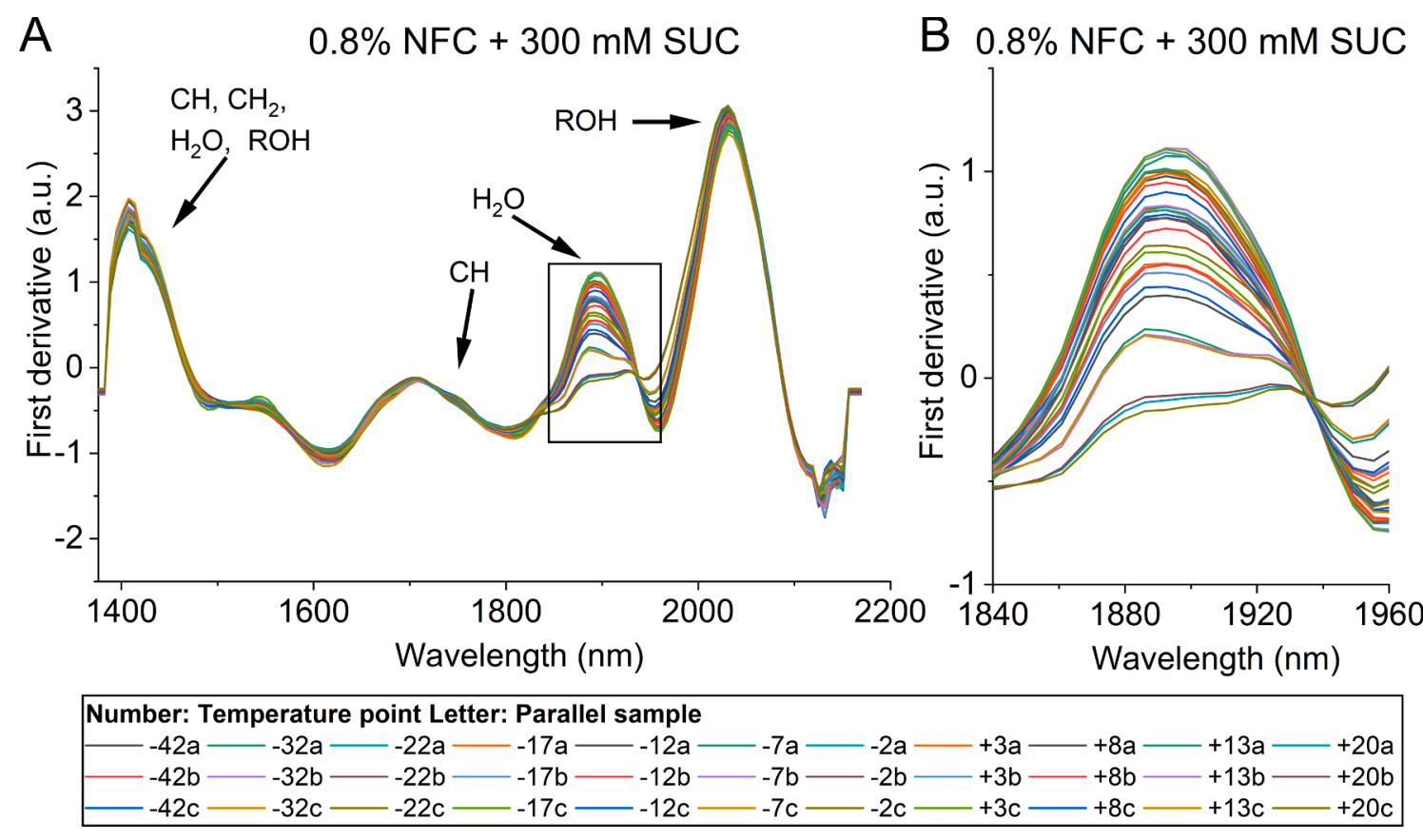

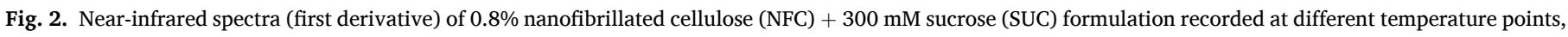
presented by the colors (legend), during the secondary drying. A) Spectra 1376-2169 nm. B) Zoomed in water region 1840-1960 nm. 
A

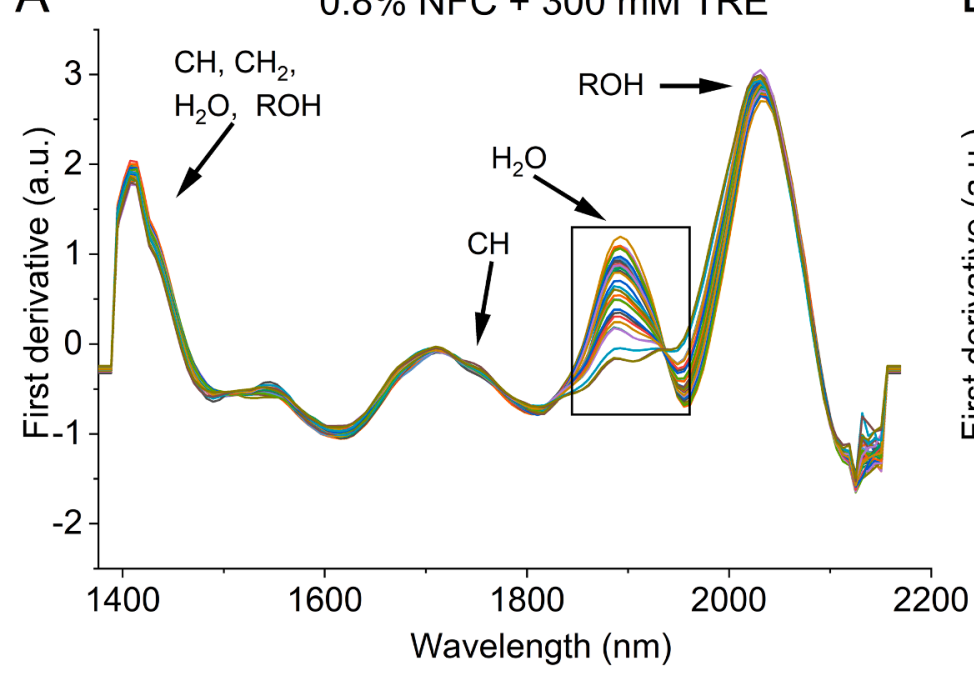

B $0.8 \% \mathrm{NFC}+300 \mathrm{mM}$ TRE

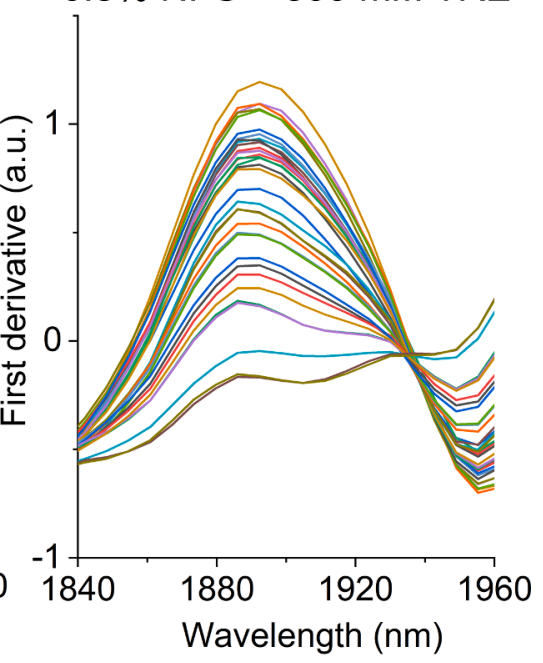

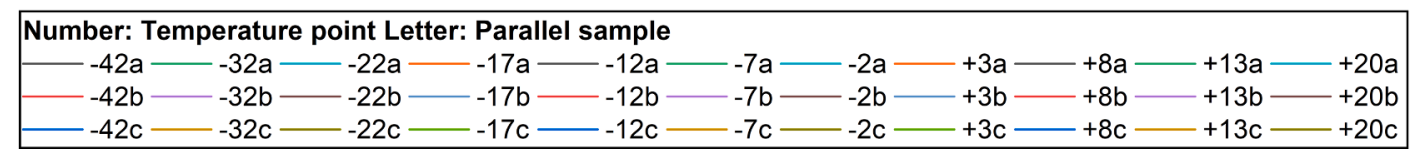

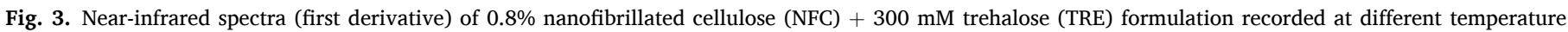
points, presented by the colors (legend), during the secondary drying. A) Spectra 1376-2169 nm. B) Zoomed in water region 1840-1960 nm.
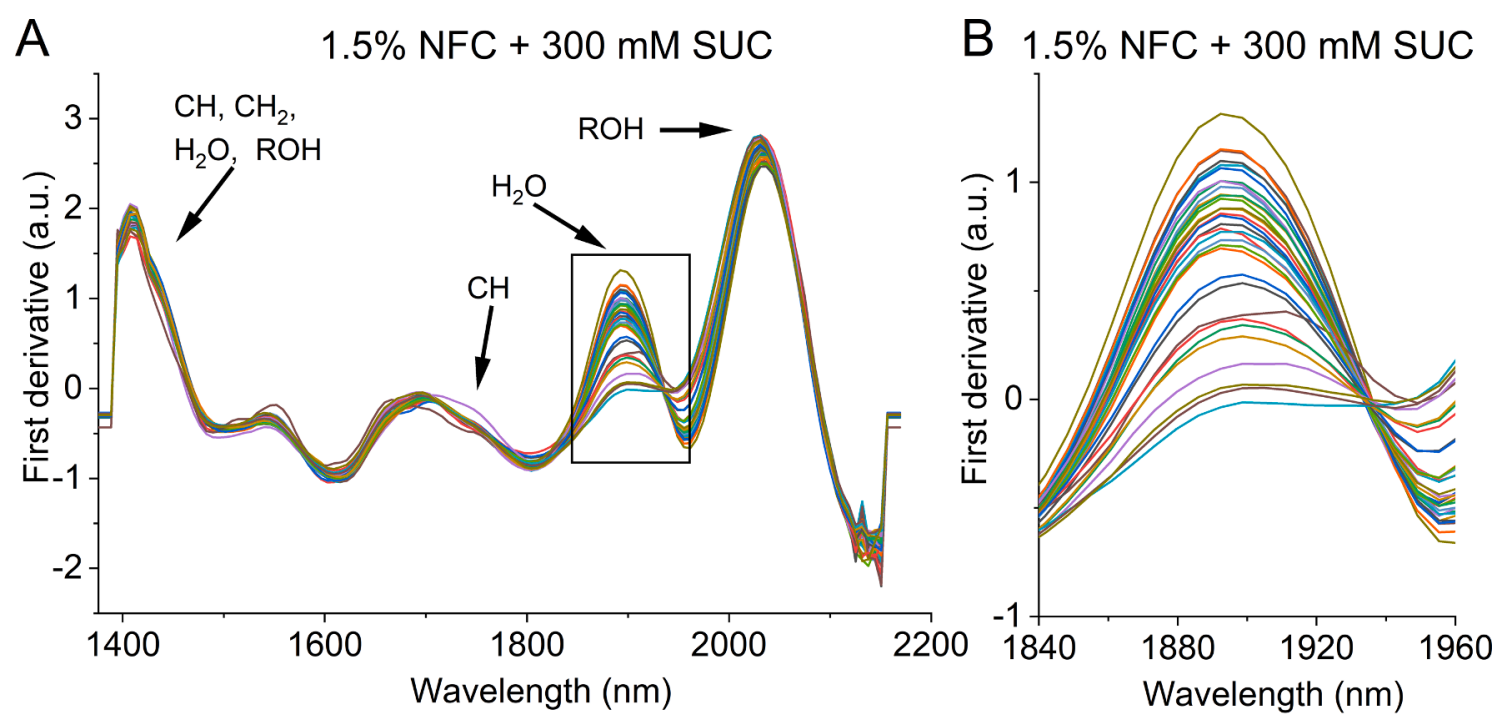

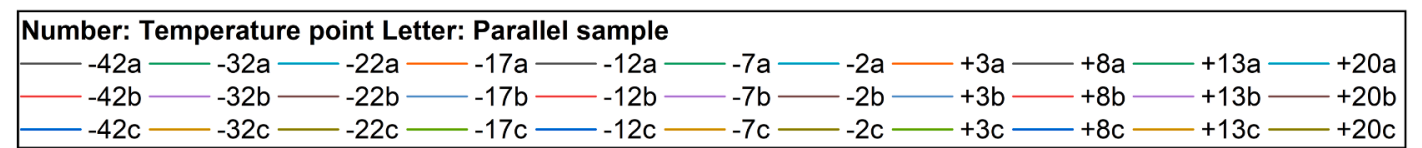

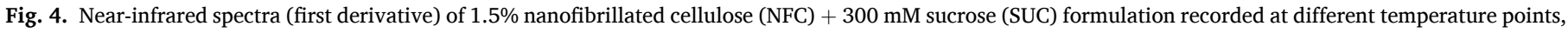
presented by the colors (legend), during the secondary drying. A) Spectra 1376-2169 nm. B) Zoomed in water region 1840-1960 nm.

energy (lower wavelength) in the local maxima at about $2000 \mathrm{~nm}$. This change was observed also with the excipients alone as presented in the Supplementary material Fig. S2-S4.

An outlier from the isosbestic point can be observed in $1.5 \%$ NFC + $300 \mathrm{mM}$ SUC formulation sample extracted at temperature point $-2{ }^{\circ} \mathrm{C}$ which was later excluded from the analysis (Fig. 4).

\subsection{Residual water content and excipients differentiated with principal component analysis}

The residual water content of each NFC hydrogel formulations with sucrose and trehalose was measured at the chosen temperature point during the secondary drying with Karl Fischer titrator. At the beginning of the whole freeze-drying process, the formulations were in hydrogel form and their water contents were $98.5 \%$ for the $1.5 \%$ NFC hydrogel formulations and $99.2 \%$ for the $0.8 \%$ NFC hydrogel formulations. The frozen water had been successfully sublimated during the primary 
A

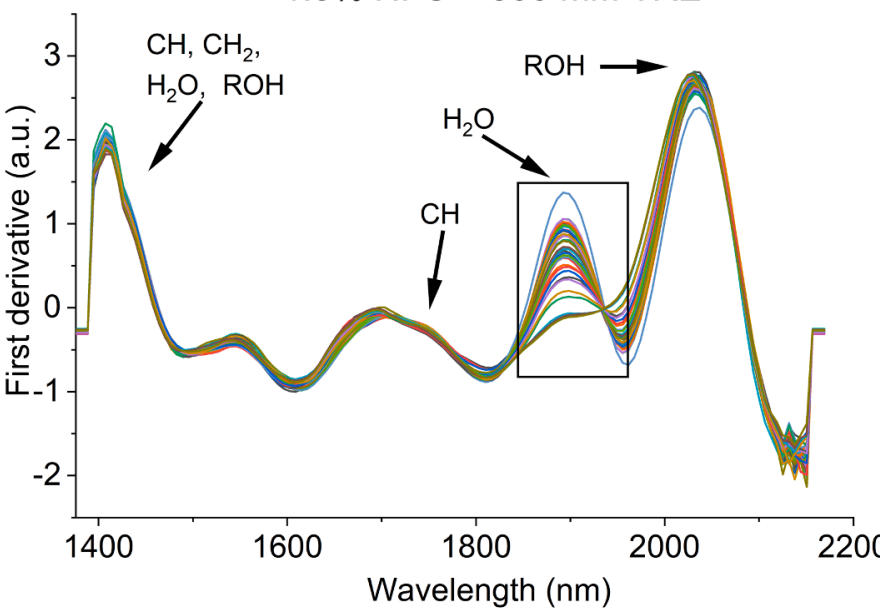

B $1.5 \% \mathrm{NFC}+300 \mathrm{mM}$ TRE

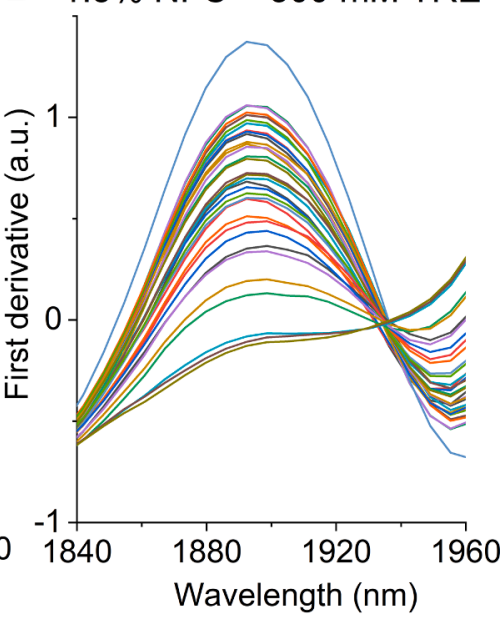

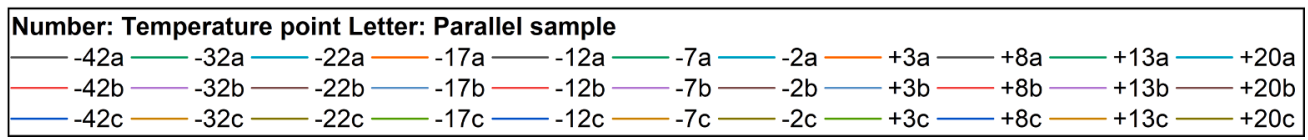

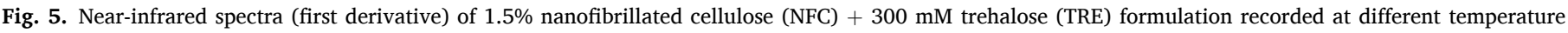
points, presented by the colors (legend), during the secondary drying. A) Spectra 1376-2169 nm. B) Zoomed in water region 1840-1960 nm.

drying and the samples did not melt when extracted from the freezedrying chamber. In addition, no collapsed samples were obtained during the secondary drying, which indicates that the residual water content was low enough and the plasticizing effect of water, i.e. decreasing glass transition $(\mathrm{Tg})$ temperature of the dry sample was low.

During the secondary drying the residual water decreased from $3.4 \%$ to $1.3 \%$ and from $3.0 \%$ to $1.0 \%$ (average, $\mathrm{n}=3$ ) for $0.8 \% \mathrm{NFC}+300 \mathrm{mM}$ SUC and $0.8 \%$ NFC $+300 \mathrm{mM}$ TRE formulations, respectively (Table 1 ). Higher residual water contents were observed with $0.8 \%$ NFC $+300 \mathrm{mM}$ SUC formulation during the whole secondary drying. A similar trend was observed with $1.5 \%$ NFC formulations at the endpoint of secondary drying: residual water content decreased from $3.1 \%$ to $1.3 \%$ and $2.9 \%$ to $1 \%$ (average, $\mathrm{n}=3$ ) for $1.5 \% \mathrm{NFC}+300 \mathrm{mM}$ SUC and $1.5 \% \mathrm{NFC}+300$ mM TRE formulations, respectively, from the beginning of the secondary drying to the endpoint. (Table 1 ). However, during the secondary drying before the endpoint, the residual water contents of $1.5 \%$ NFC SUC and TRE formulations were nearly identical. Interestingly, all formulations showed increased residual water contents at the beginning of the secondary drying when the temperature was increased, with the highest residual water contents at temperature points of $-17{ }^{\circ} \mathrm{C}$ and $-12{ }^{\circ} \mathrm{C}$ (Fig. 6A). Similar results were obtained with the $300 \mathrm{mM}$ SUC and 300 mM TRE control samples without NFC hydrogel (Supplementary information, Fig. S5). Relatively stable and slightly increasing water contents at the beginning of secondary drying can be explained by the high temperature of the sample (Supplementary information, Fig. S1). The temperature of the sample was higher than the shelf temperature which means that no energy was conducted to the sample resulting in stalled desorption of water. The slight increase of water content can result from the leakage of the extractor door during the sample extraction.

All samples collected during the secondary drying were used for the
PCA and a spectral region of 1376-2169 nm was used. PCA scores plot confirmed the trend of measured residual water contents and the increase of residual water contents during the first temperature points (from $-42{ }^{\circ} \mathrm{C}$ to $-17{ }^{\circ} \mathrm{C} /-12{ }^{\circ} \mathrm{C}$ ) (Fig. 6B). The scores of the samples extracted at $-42{ }^{\circ} \mathrm{C}$ were near 0 on the principal component (PC) 1 axis (red marks, water $3-4 \%$ ), then the score shifted to the negative side for samples extracted at $-32{ }^{\circ} \mathrm{C}$ to $-12{ }^{\circ} \mathrm{C}$ (blue marks, water $>4 \%$ ) and when the secondary drying process continued, the scores shifted to the positive side i.e. for the samples extracted at temperature points from $-12{ }^{\circ} \mathrm{C}$ to $+20{ }^{\circ} \mathrm{C}$ water decreased from $4 \%$ to below $2 \%$ (red marks water $3-4 \%$, green marks water $2-3 \%$, black marks water $<2 \%$ ). NFC concentrations, $0.8 \%$ and $1.5 \%$, clustered according to PC2 on the positive and negative sides, respectively. PC3 explained the variation caused by different excipients (Fig. 6C). NFC formulations are clustered by their fiber concentration (PC2) and by their excipients (PC3) into different groups.

Loading plot (Fig. 6D) shows high weights for PC1 on 1820-1940 nm and 1940-2050 nm for negative and positive load, respectively. When loading plots are compared with the spectra, PC1 corresponds to residual water contents and samples with the highest residual water content have the highest score on PC1. PC2 has a high load in 2000-2100 nm which corresponds to the OH-band of the NIR spectrum. The difference can be explained by the different ratios of excipient and NFC fibers. $0.8 \%$ NFC formulations have higher absorption in that region (See Figs. 2-5) which can indicate more unbound sugars i.e. sucrose and trehalose are present. Contrary, with $1.5 \%$ NFC formulations the ratio is lower, and potentially more hydrogen bonds between the sugars and NFC fibers have formed resulting in less free hydroxyl groups, and thus, lower absorption on that region is observed. PC2 has negative loading at $1600-1700 \mathrm{~nm}$ and a difference is observed in spectra of $0.8 \%$ and $1.5 \%$

Table 1

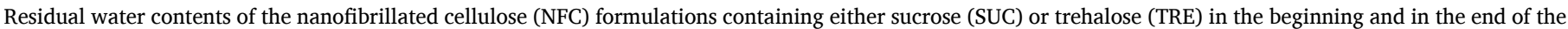
secondary drying.

\begin{tabular}{|c|c|c|}
\hline Formulation & Residual water content in the beginning of the secondary drying & Residual water content in the end of the secondary drying \\
\hline $0.8 \% \mathrm{NFC}+300 \mathrm{mM}$ SUC & $3.4 \%$ & $1.3 \%$ \\
\hline $0.8 \% \mathrm{NFC}+300 \mathrm{mM}$ TRE & $3.0 \%$ & $1.0 \%$ \\
\hline $1.5 \% \mathrm{NFC}+300 \mathrm{mM}$ SUC & $3.1 \%$ & $1.3 \%$ \\
\hline $1.5 \% \mathrm{NFC}+300 \mathrm{mM}$ TRE & $2.9 \%$ & $1 \%$ \\
\hline
\end{tabular}


A

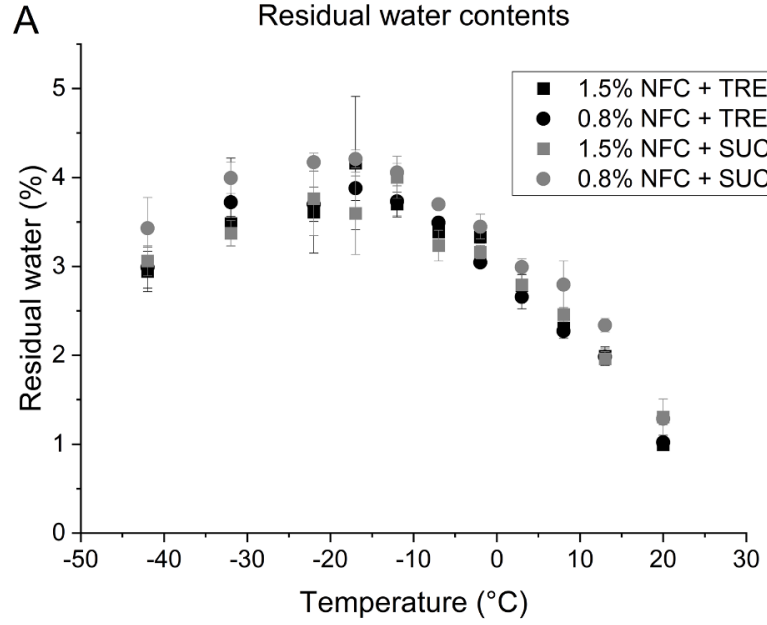

C

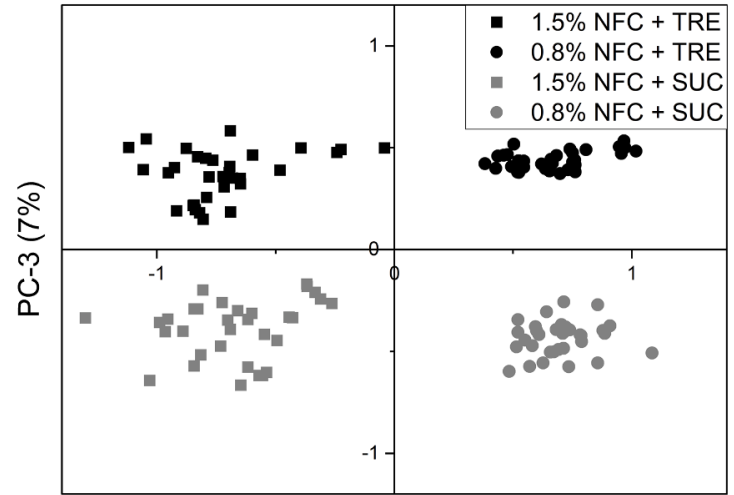

PC-2 (20\%)
B PC-1 and PC-2 of all formulations
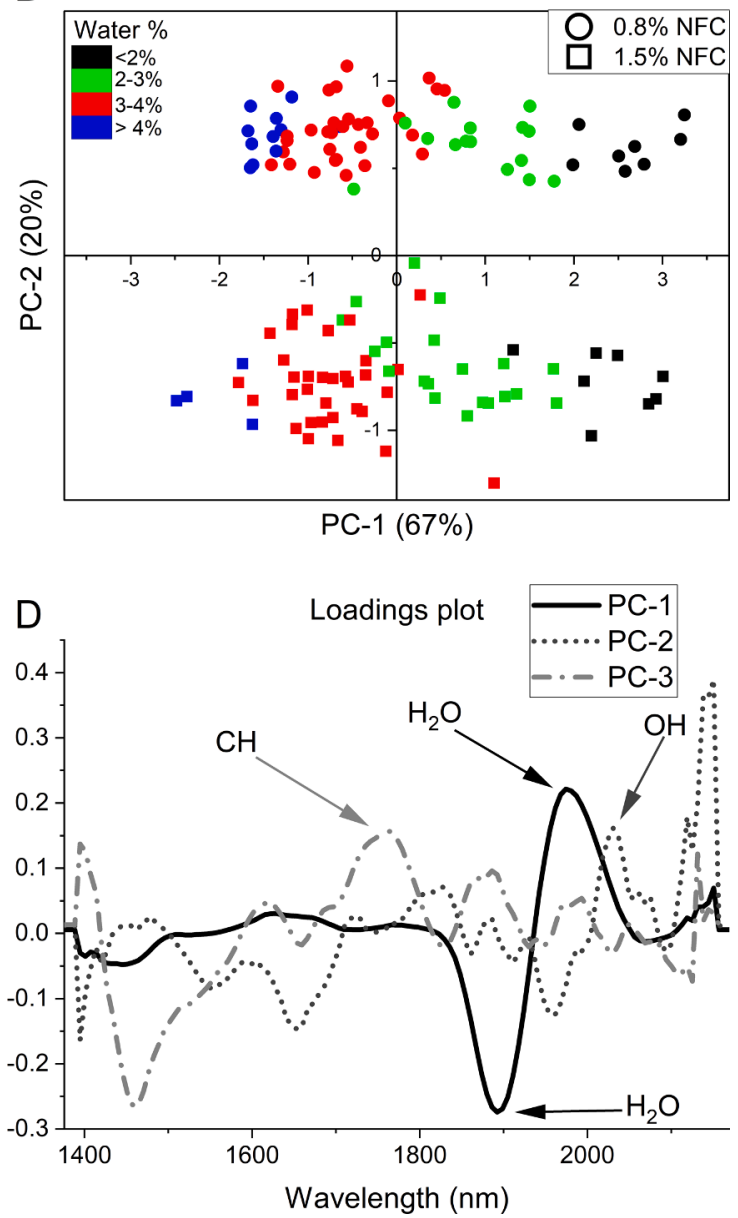

Fig. 6. A) Residual water content of $0.8 \%$ (circle) and $1.5 \%$ (square) nanofibrillated cellulose (NFC) hydrogel formulations with $300 \mathrm{mM}$ trehalose (TRE) and 300 $\mathrm{mM}$ sucrose (SUC) formulations indicated with black and gray, respectively. Residual water contents of all NFC hydrogel formulations first increased during the secondary drying and then decreased. At the final temperature point, i.e. at the end of the secondary drying, the same residual water contents were obtained for SUC and TRE formulations regardless of the NFC fiber concentration. B) Scores plot of principal component (PC) 1 and PC2 of principal component analysis (PCA) of all NFC formulations. PC1 explains $67 \%$ of variation which results from the residual water content. PC2 explains $20 \%$ of the variation which results from the different NFC concentrations. $0.8 \%$ NFC (circle) formulations clustered on positive side of PC2 and 1.5\% NFC (square) formulations on the negative side. Residual water decreases from the left to the right (PC1). C) Scores plot of PC2 and PC3 of PCA analysis of all NFC formulations. PC2 explains $20 \%$ of the variation which resulted from the different NFC concentrations. PC3 explains 7\% of the variation which resulted from the differences between excipients. $1.5 \%$ NFC $+300 \mathrm{mM}$ TRE (black squares) clustered on upper left sector, $1.5 \% \mathrm{NFC}+300 \mathrm{mM}$ SUC (gray squares) on the lower left sector, $0.8 \% \mathrm{NFC}+300 \mathrm{mM}$ TRE (black circles) on the upper right sector, and $0.8 \% \mathrm{NFC}+300 \mathrm{mM}$ SUC on the lower right sector (gray circles). D) Loading plot of 1.5\% NFC formulations. PC-1 main regions are $1820-1940 \mathrm{~nm}$ (negative load) and 1940-2050 nm (positive load), PC-2 main regions are 2000-2100 nm (positive load) and 1600-1700 (negative load), and PC-3 main regions are 1420-1600 nm (negative load) and 1700-1800 nm (positive load).

NFC formulations on that area. The local maxima of $1.5 \%$ NFC formulation's spectra are at a lower wavelength i.e. higher energy on that area.

A high positive load for PC3 is observed at $1700-1800 \mathrm{~nm}$. $1700-1800 \mathrm{~nm}$ is the absorption region of the $\mathrm{CH}$-group (Fig. 6). PC3 can be explained by the different number of $\mathrm{CH}$-groups of the excipients (See Fig. 1 molecular structures). Trehalose molecules have $10 \mathrm{CH}$ groups while sucrose molecules 8 , and this can explain the slight difference between the spectra of different formulations. The same difference can be observed also with the pure excipients without NFC hydrogel (Supplementary information, Fig. S2-S4, S6).

\subsection{Partial least square models predict residual water content of NFC formulations accurately}

PLS regression models were built to predict the residual water content of NFC hydrogel formulations with excipients during the secondary drying. The training set was validated with leave-one-out cross-validation, after which cross-validation was performed with a larger set of samples.

The PLS models predict accurately the residual water content of a multicomponent biomaterial formulation of NFC hydrogel with sucrose or trehalose (Fig. 7). The PLS model was trained with every other spectrum of each formulation collected at different temperature points (Fig. 7A) and the remaining spectra were used as the cross-validation set to evaluate the model. The $\mathrm{R}^{2}$ value of 0.907 and RMSECV value of $0.259 \% \mathrm{w} / \mathrm{w}$ were obtained for the prediction of residual water contents of the cross-validation set (Fig. 7B). The average RSECV of the crossvalidation set is $9.64 \%$ and the bias of the model is 0.020 percent points indicating a random distribution of points around the regression line. Most of the variation can be explained by factor- 1 , which explains $68 \%$ of the variation in the spectral data and $94 \%$ of the variance in the residual water. Factor- 2 explains $19 \%$ of the variation in the spectral data and $1 \%$ of the variance in the residual water contents. The loading plot of factors (Fig. 7C) indicates clearly that the absorption band of water (1820-1940 nm) impacts mostly on factor-1 and the absorption band at 2000-2100 nm impacts on factor-2. This showed clearly, that 
A

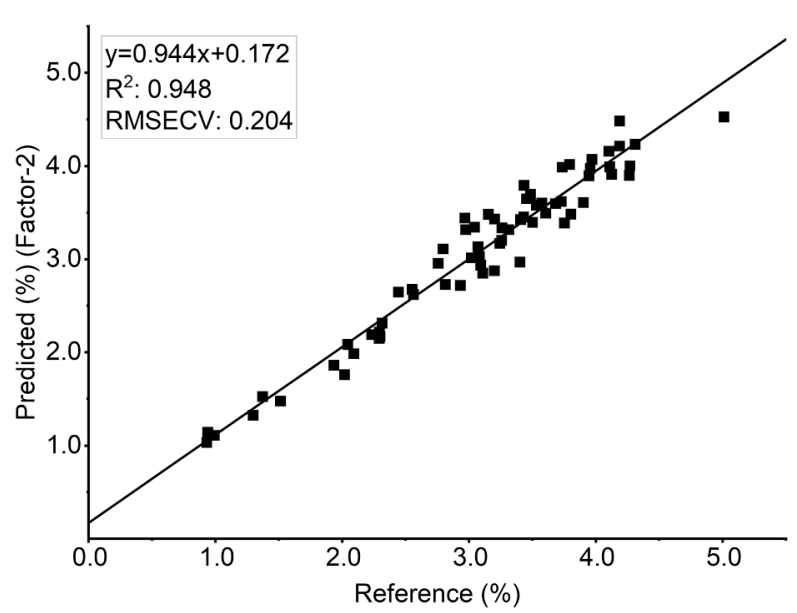

C Loading plot of factor-1 and factor-2

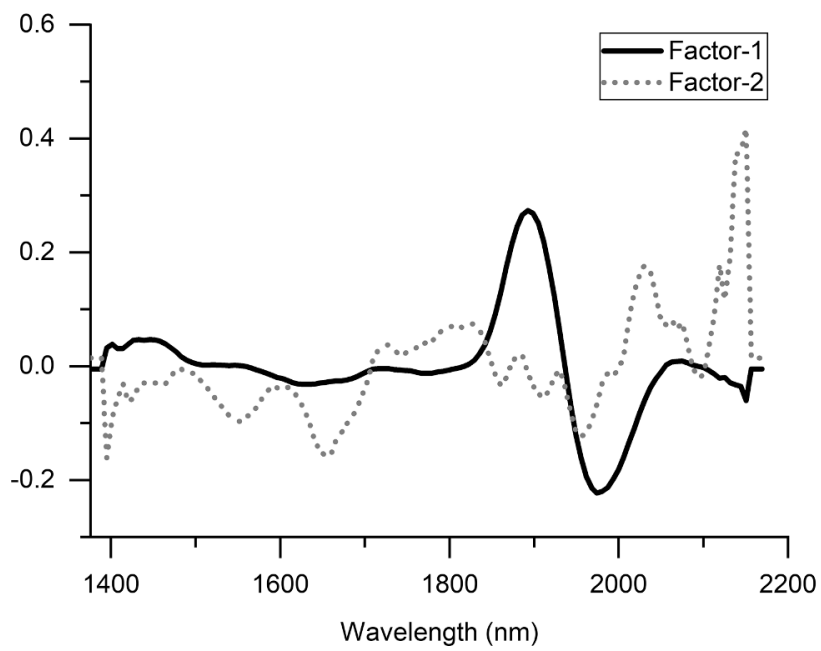

\section{B Predicted residual water content of all formulations}

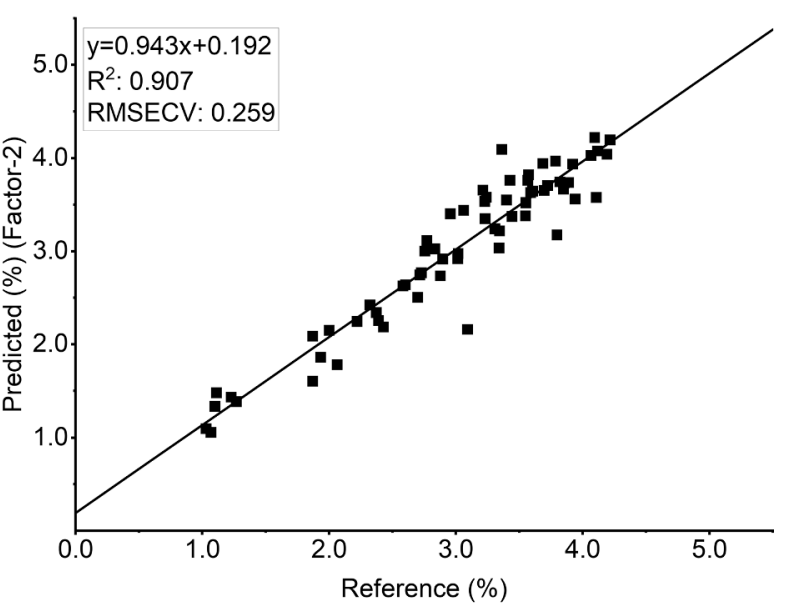

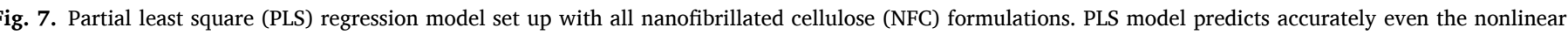

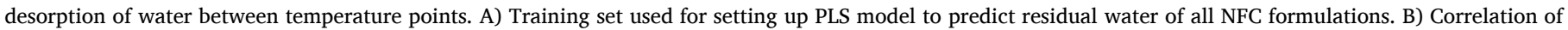

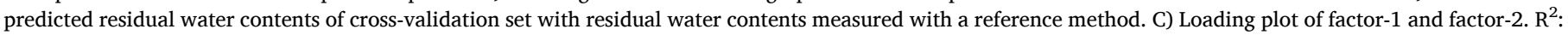
Coefficient of determination. RMSECV: Root mean square error of prediction.

the NIR system predicts highly accurately residual water content of multicomponent biomaterial formulations.

We also evaluated the potential of PLS models to predict residual water if the same excipients were used to train the model, but the original NFC fiber concentration was different. The objective of this evaluation was to study the potential to generalize the PLS regression model to different concentrations of NFC hydrogel. In addition, the formulations with different NFC concentrations were used as external validation sets because they were freeze-dried as another batch. For sucrose, when $0.8 \% \mathrm{NFC}+300 \mathrm{mM}$ SUC was used as the training set and $1.5 \% \mathrm{NFC}+300 \mathrm{mM}$ SUC as the external validation set, the $\mathrm{R}^{2}$ value of 0.850 and RMSEP value of $0.310 \% \mathrm{w} / \mathrm{w}$ were obtained. The bias of the model was -0.137 percent points and average RSEP $11.8 \%$ indicating satisfactory prediction with 2-factors in prediction. For trehalose formulations, more accurate predictions were obtained. When $0.8 \% \mathrm{NFC}+$ $300 \mathrm{mM}$ TRE was used as the training set and 1.5\% NFC + $300 \mathrm{mM}$ TRE as the external validation set, $0.953 \mathrm{R}^{2}$ and $0.194 \% \mathrm{w} / \mathrm{w}$ RMSEP were obtained with only 1 -factor in the prediction. The bias of the model was 0.046 percent points and RSEP $7.12 \%$. Data are shown in Supplementary information Fig. S7 and PLS models of individual formulations are shown in Supplementary information Fig. S8.

\subsection{Highly porous NFC aerogels were obtained by freeze-drying}

SEM, $\mu$-CT, and PXCT were used first, to ensure the successful formation of aerogels and second, to observe any differences in the morphology, porosity, or electron densities of NFC aerogels. NFC hydrogel freeze-dried with sucrose or trehalose formed a highly porous aerogel. SEM micrographs of $0.8 \% \mathrm{NFC}+300 \mathrm{mM}$ SUC and $1.5 \% \mathrm{NFC}+$ $300 \mathrm{mM}$ SUC both show a regular porous aerogel structure (Fig. 8). No difference between SUC and TRE formulations can be judged from the SEM micrographs (See SEM micrographs of trehalose formulations in Supplementary information, Fig. S9). Closed pores appear to be more prevalent in $1.5 \%$ NFC formulations than in $0.8 \%$ formulations independently of the excipient. In addition, fewer fiber structures can be observed in the $1.5 \%$ NFC formulations than in the $0.8 \%$ NFC formulations. However, both $1.5 \%$ and $0.8 \%$ NFC formulations show clear aerogel morphology. NFC control samples i.e. pure NFC without excipients showed also porous structure (Supplementary information, Fig. S9). However, the texture of the freeze-dried pure NFC was spongy and gummy, compared to the more rigid texture of freeze-dried NFC aerogels with sucrose and trehalose. The $\mu$-CT results revealed that the porosity of $1.5 \% \mathrm{NFC}+300 \mathrm{mM}$ SUC are $91.9 \%$ and $89.6 \%$ for replicates 1 and 2 , respectively, and porosity of $0.8 \% \mathrm{NFC}+300 \mathrm{mM}$ SUC aerogel $86.3 \%$ and $89.9 \%$, for replicates 1 and 2 , respectively, of the whole 


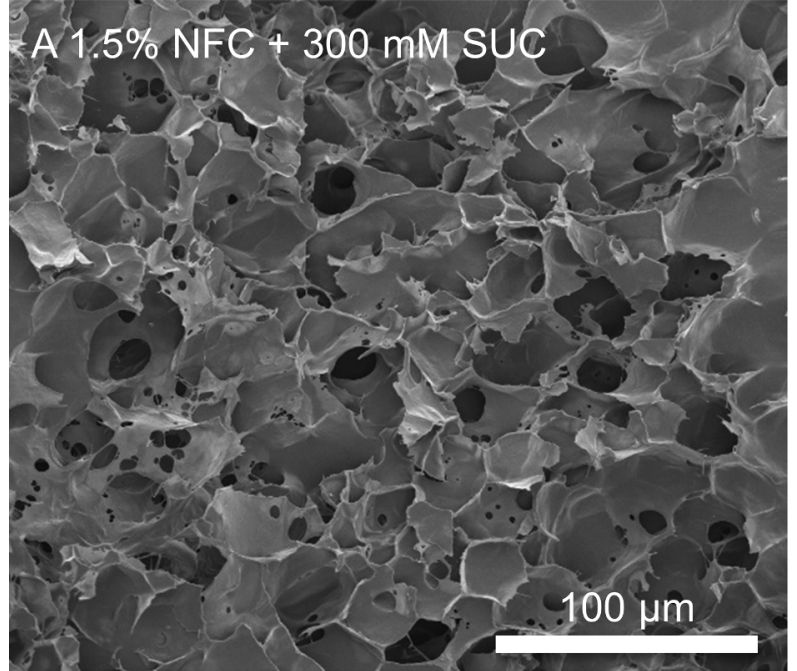

C

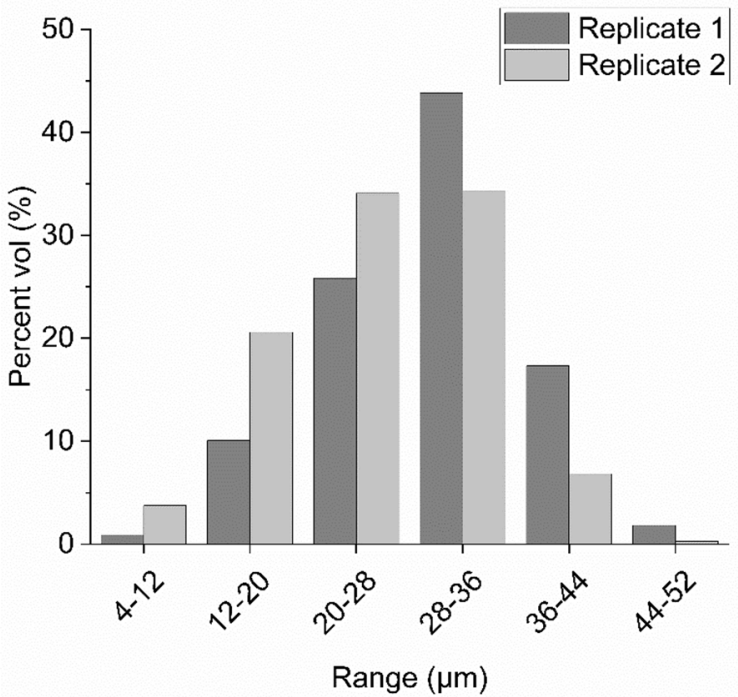

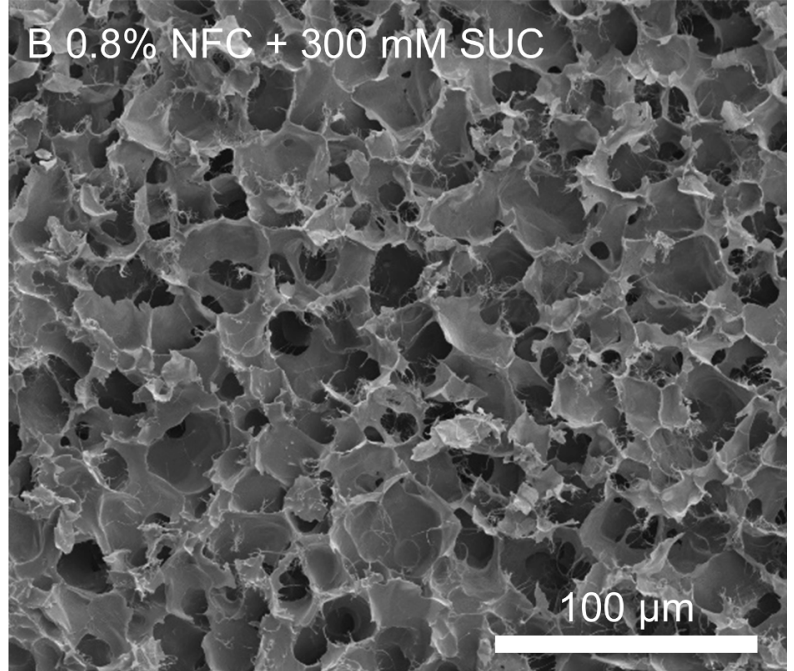

$0.8 \%$ NFC $0300 \mathrm{mM}$ SUC

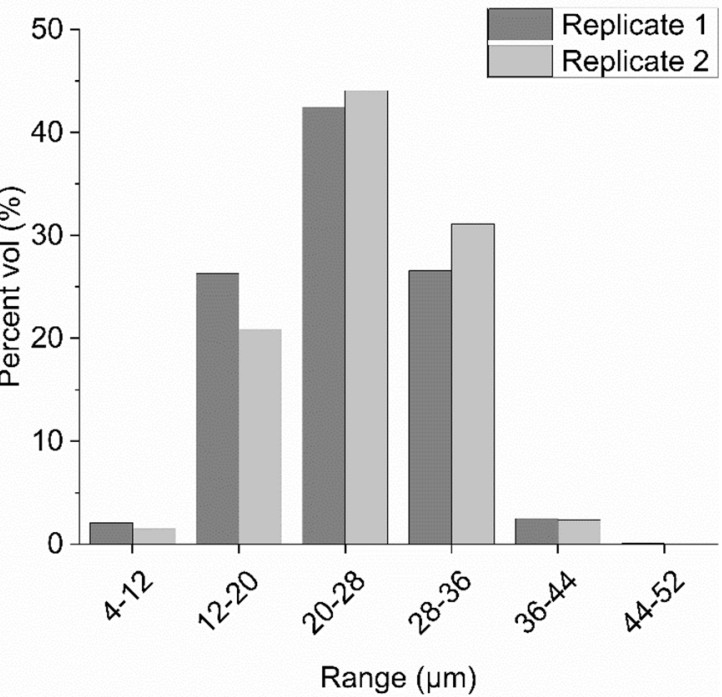

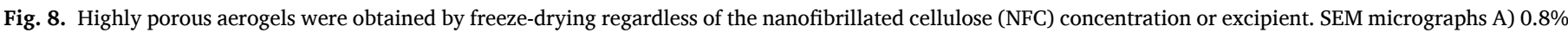

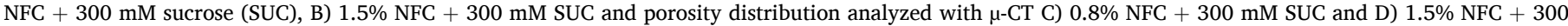
$\mathrm{mM}$ SUC.
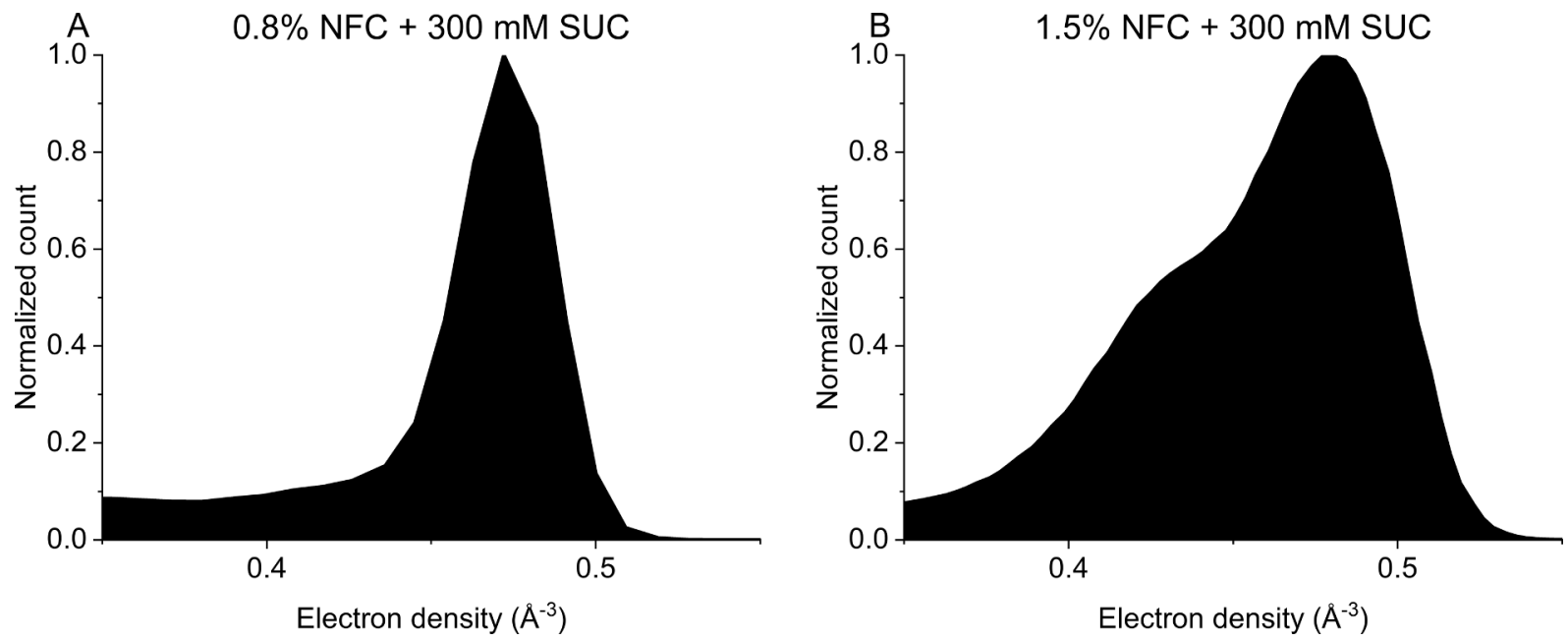

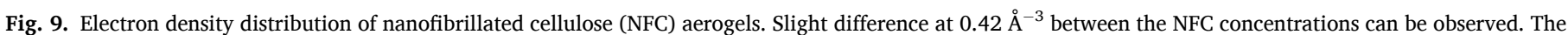

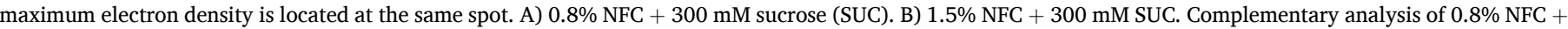
$300 \mathrm{mM}$ SUC is shown also in Koivunotko et al., 2021. 
volume. The pore diameter distribution is shown in Fig. 8. Interestingly, slightly more variation is observed with the formulation of $1.5 \%$ NFC. Similar results were obtained for the NFC formulations with trehalose (Supplementary Information, Fig S10 and slices of images used for the analysis in Fig S8). When $1.5 \%$ NFC and $0.8 \%$ NFC were freeze-dried without excipients, no variation was observed in the porosity distribution regardless of the fiber concentration (Supplementary Information, Fig. S10). Taken together, freeze-drying yielded highly porous NFC aerogels with honeycomb-like morphology.

PXCT was used to evaluate the electron density of freeze-dried NFC aerogels with sucrose. The most prevalent electron density for $0.8 \%$ $\mathrm{NFC}+300 \mathrm{mM}$ SUC and $1.5 \% \mathrm{NFC}+300 \mathrm{mM}$ SUC formulations was $0.47 \AA^{-3}$ and $0.48 \AA^{-3}$, respectively. Similar results were obtained for the $1.5 \%$ NFC control sample, for which $0.48 \AA^{-3}$ was the most prevalent electron density. The electron density distribution of $1.5 \%$ NFC +300 mM SUC and $0.8 \%$ NFC $+300 \mathrm{mM}$ SUC formulations had minor differences. The $1.5 \% \mathrm{NFC}+300 \mathrm{mM}$ SUC formulation showed a shoulder at $0.42 \AA-3$ which was missing in $0.8 \%$ NFC +300 mM SUC formulations (Fig. 9). Analyzing the tomographic slices more closely revealed that the $0.42 \AA-3$ electron density was mostly on the surfaces of the pore walls, while the higher $0.48 \AA-3$ electron density was observed in the interior of the aerogel walls. Nanoporosity was observed in two specimens of $1.5 \% \mathrm{NFC}+300 \mathrm{mM}$ SUC and in none of the $0.8 \% \mathrm{NFC}+300 \mathrm{mM}$ SUC. The electron density distribution of the $1.5 \%$ NFC control and slices of PTCT analysis are presented in Supplementary Information, Fig. S11. Complementary results of $0.8 \%$ NFC $+300 \mathrm{mM}$ SUC and control NFC samples are also presented in Koivunotko et al., 2021.

\section{Discussion}

Biophotonic in-line process analytical technology will be needed ever increasingly in the freeze-drying of biomaterials for aerogel fabrication. This study aimed to characterize the manufacturing of biomaterial aerogels with NIR spectroscopy. We showed that NIR spectroscopy detects the residual water content, a critical quality attribute of freezedried aerogels, of multicomponent NFC aerogel formulations during secondary drying of freeze-drying, and we built PLS regression models which predict accurately their residual water content. In addition, we analyzed the second critical quality attribute of aerogels, porosity, of NFC aerogels fabricated by freeze-drying thoroughly with SEM and $\mu$-CT, and the morphology and electron densities were further analyzed by PXCT.

Previously NIR prediction has been thought to be dependent on the concentrations of the excipients and active pharmaceutical ingredients (Kauppinen et al., 2013; Lin and Hsu, 2002), i.e., each formulation needs to be validated independently. Recently, Clavaud et al. (2020) used a Design of Experiment approach to cover a large range of different samples and process conditions to allow the application of PLS models for residual water content prediction for a wider range of formulations. In our study, we used as a training set two different NFC concentrations and two different excipients and obtained a set of samples to cover a wider area of formulations. In addition, we set up the PLS models that predict residual water content regardless of the NFC concentration by using different NFC concentrations in the training set than in the external validation set. Furthermore, the PLS models predicted accurately the nonlinear behavior i.e. first the increase and then the decrease, of residual water, which indicates that NIR is suitable as PAT also to monitor nonlinear and heterogeneous drying. The observed nonlinear behavior of the residual water contents during the secondary drying result potentially due to the leakage of the sampling door. NIR spectroscopy has been shown also previously to detect the heterogenous drying with fluid bed dryers (Green et al., 2005). Taken together, using a multipoint NIR system to monitor aerogel fabrication at different locations of the freeze-dryer tray would benefit in shortening the cycle and avoiding the formation of spongy cryogels (García-González et al., 2021; Kauppinen et al., 2013).
NIR was not only accurate in detecting the residual water contents, but we could separate NFC formulations with different excipients from each other with PCA analysis of NIR spectra despite the similar chemical structures of cellulose monomers (glucose), trehalose (glucose-glucose), and sucrose (glucose-fructose). PC1 corresponded to the residual water content and distributed the samples according to their residual water content on the scores plot which is already a well-known application (Clavaud et al., 2020; Kamat et al., 1989; Kauppinen et al., 2013). PC2 separated samples according to their NFC fiber amount from the absorbance wavelengths $1600-1700 \mathrm{~nm}$ and $2050-2100 \mathrm{~nm}$. We explained the difference by different ratios of NFC fibers and excipients resulting in different numbers of OH-groups. PC3 distinguished trehalose and sucrose samples from each other. This separation compromises mostly from the 1st overtone of the CH-group at wavelength 1700-1800 $\mathrm{nm}$ (Weyer and Lo, 2006). Trehalose and sucrose have different numbers of $\mathrm{CH}$-groups which can result in differences in that absorbance region. Applying these results further would allow monitoring not only the residual water contents and proper drying of aerogel, but potentially also the proper embedding of active pharmaceutical ingredients, whether it be small molecules or therapeutic proteins, into aerogel. However, these applications require further optimization, and no straight translation of the obtained results can be extrapolated to other formulations.

Porosity is recognized as a critical quality attribute of aerogels. In this study, we evaluated the porosity of aerogels with SEM, $\mu$-CT, and PXCT. PXCT provided information also about the electron density distribution. All NFC formulations formed highly porous regular aerogels with a firm texture and only slight morphological differences were observed between $1.5 \%$ NFC and $0.8 \%$ NFC formulations in the SEM micrograph. Contrary, the texture of freeze-dried NFC samples without excipients was spongy despite the morphology evaluated from SEM micrographs resembling the NFC aerogels with excipients. This correlates with our previous results on how excipients facilitated the successful reconstitution of freeze-dried NFC aerogel to NFC hydrogel (Koivunotko et al., 2021). No difference was observed between the SEM micrographs of SUC and TRE formulations. Furthermore, based on the similar rate of decrease of residual water during the secondary drying we can conclude that the overall surface area is the same for each sample (Oddone et al., 2017; Pikal et al., 1990). This is supported by the porosity analyses performed with $\mu$-CT. The overall porosity of freezedried NFC aerogels was similar, yet, slightly more variation was observed in the porosity distribution of parallel samples of $1.5 \%$ NFC formulations. This can contribute to the minor heterogeneity in the water decrease rate which was observed between parallel samples with $1.5 \%$ NFC formulations. Interestingly, $0.8 \%+300 \mathrm{mM}$ SUC and $1.5 \%$ $\mathrm{NFC}+300 \mathrm{mM}$ SUC formulations showed also minor difference in the electron density distribution. The expected electron densities of cellulose and sucrose are $0.53 \AA^{-3}$ and $0.51 \AA^{-3}$, respectively (Diaz et al., 2012) (See Supplementary for calculations). When looking at the electron density histograms, it is evident that only a minor part of the sample resembled those electron densities. However, the calculated density is for a fully crystalline structure and the product is a partially amorphous material, and thus the density is lower than that of the crystalline material. The calculated electron density of amorphous sucrose is $0.49 \AA^{-3}$. This corresponds very well to the most prevalent electron density value observed in the NFC with sucrose and the shoulder observed in the electron density histogram suggests that in the $1.5 \% \mathrm{NFC}+300 \mathrm{mM}$ formulation regions with different densities are present. These different regions could also partly explain the variation in the porosity observed with $\mu$-CT and variation in the decreased rate of water during the secondary drying. The difference potentially originates from the different ratios of NFC fibers and sucrose molecules resulting in variation in the number of unbound sucrose molecules as the same difference was observed in the NIR spectra. The higher electron density in the center of the walls compared to the wall surfaces can be explained by the freezing process: During the freezing step, the polymers concentrate outside the ice crystals and the ice, which is later sublimated, forms the pores 
(Grenier et al., 2019). Potentially, during the freezing, the polymers and the excipients pack tighter to the aerogel wall interiors which is then observed as different electron densities. However, no absolute conclusions can be made without further assessment.

\section{Conclusions}

Nanofibrillated cellulose hydrogel formulations were successfully freeze-dried, and highly porous aerogels were obtained. NIR proved to be a powerful tool for monitoring the residual water content of biomaterials during freeze-drying and to separate different excipients used in the formulations from each other. We showed that PLS models can be generalized to measure the residual water content of NFC formulations with varying fiber concentrations and excipients facilitating wider utilization of NIR as process analytical technology in the freeze-drying of biomaterials. We believe that NIR is necessary equipment to be added as a common practice to the freeze-drying of biomaterials and that these results can be further applied for the manufacturing of biomaterial aerogels by freeze-drying.

\section{Credit authorship contribution statement}

Arto Merivaara: Conceptualization, Methodology, Formal analysis, Investigation, Writing - original draft, Writing - review \& editing, Supervision, Funding acquisition. Jere Kekkonen: Conceptualization, Methodology, Formal analysis, Investigation, Writing - review \& editing, Supervision, Funding acquisition. Julia Monola: Conceptualization, Methodology, Formal analysis, Investigation, Writing - review \& editing, Funding acquisition. Elle Koivunotko: Conceptualization, Formal analysis, Investigation, Supervision, Funding acquisition. Marko Savolainen: Methodology, Resources, Writing - review \& editing. Tuomo Silvast: Methodology, Formal analysis, Investigation, Writing review \& editing. Kirsi Svedström: Methodology, Resources, Writing review \& editing. Ana Diaz: Methodology, Investigation, Resources, Writing - review \& editing. Mirko Holler: Methodology, Investigation, Resources, Writing - review \& editing. Ossi Korhonen: Conceptualization, Methodology, Formal analysis, Resources, Supervision, Writing review \& editing. Marjo Yliperttula: Conceptualization, Formal analysis, Resources, Supervision, Funding acquisition, Writing - original draft, Writing - review \& editing. Sami Valkonen: Conceptualization, Methodology, Formal analysis, Investigation, Supervision, Writing original draft, Writing - review \& editing.

\section{Declaration of Competing Interest}

The authors declare that they have no known competing financial interests or personal relationships that could have appeared to influence the work reported in this paper.

\section{Acknowledgements}

The authors acknowledge Electron Microscopy Unit of the Institute of Biotechnology, University of Helsinki for providing laboratory facilities.

AM acknowledges funding from Emil Aaltonen Foundation grants no. 200141N and 210137N; UPM Business Finland (BF) GD-96 -project (MY); EVE projects no. 2581/31/2018 (MY, SV) and no. 1842/31/2019 (MY, SV) are acknowledged. AM, JK, JM, and OK acknowledge funding from NordForsk for the Nordic University Hub project \#85352 (Nordic POP, Patient Oriented Products). This research is financially supported by Academy of Finland GeneCellNano Flagship (grant 337430) (MY).

\section{Appendix A. Supplementary material}

Supplementary data to this article can be found online at https://doi. org/10.1016/j.ijpharm.2022.121581.

\section{References}

Athamneh, T., Amin, A., Benke, E., Ambrus, R., Leopold, C.S., Gurikov, P., Smirnova, I., 2019. Alginate and hybrid alginate-hyaluronic acid aerogel microspheres as potential carrier for pulmonary drug delivery. J. Supercrit. Fluids 150, 49-55. https://doi.org/10.1016/j.supflu.2019.04.013.

de Beer, T., Burggraeve, A., Fonteyne, M., Saerens, L., Remon, J.P., Vervaet, C., 2011. Near infrared and Raman spectroscopy for the in-process monitoring of pharmaceutical production processes. Int. J. Pharm. 417 (1-2), 32-47. https://doi. org/10.1016/j.ijpharm.2010.12.012.

Blasi-Romero, A., Palo-Nieto, C., Sandström, C., Lindh, J., Strømme, M., Ferraz, N., 2021. In vitro investigation of thiol-functionalized cellulose nanofibrils as a chronic wound environment modulator. Polymers 13 (2), 249. https://doi.org/10.3390/ polym13020249.

Brougham, C.M., Levingstone, T.J., Shen, N., Cooney, G.M., Jockenhoevel, S., Flanagan, T.C., O'Brien, F.J., 2017. Freeze-drying as a novel biofabrication method for achieving a controlled microarchitecture within large, complex natural biomaterial scaffolds. Adv. Healthcare Mater. 6 (21), 1700598. https://doi.org/ 10.1002/adhm.201700598.

Cabeza, L., Perazzoli, G., Peña, M., Cepero, A., Luque, C., Melguizo, C., Prados, J., 2020. Cancer therapy based on extracellular vesicles as drug delivery vehicles. J. Control. Release 327, 296-315. https://doi.org/10.1016/j.jconrel.2020.08.018.

Cascone, S., Lamberti, G., 2020. Hydrogel-based commercial products for biomedical applications: A review. Int. J. Pharm. 573, 118803. https://doi.org/10.1016/j. ijpharm.2019.118803.

Chen, Y., Zhou, L., Chen, L., Duan, G., Mei, C., Huang, C., Han, J., Jiang, S., 2019. Anisotropic nanocellulose aerogels with ordered structures fabricated by directional freeze-drying for fast liquid transport. Cellulose 26 (11), 6653-6667. https://doi. org/10.1007/s10570-019-02557-z.

Clavaud, M., Lema-Martinez, C., Roggo, Y., Bigalke, M., Guillemain, A., Hubert, P., Ziemons, E., Allmendinger, A., 2020. Near-infrared spectroscopy to determine residual moisture in freeze-dried products: model generation by statistical design of experiments. J. Pharm. Sci. 109 (1), 719-729. https://doi.org/10.1016/j. xphs.2019.08.028.

Diaz, A., Trtik, P., Guizar-Sicairos, M., Menzel, A., Thibault, P., Bunk, O., 2012 Quantitative X-ray phase nanotomography. Phys. Rev. B 85 (2), 020104. https://doi. org/10.1103/PhysRevB.85.020104.

Franks, F., Auffret, T., 2008. Freeze-drying of Pharmaceuticals and Biopharmaceuticals. The Royal Society of Chemistry, London.

García-González, C.A., Sosnik, A., Kalmár, J., de Marco, I., Erkey, C., Concheiro, A., Alvarez-Lorenzo, C., 2021. Aerogels in drug delivery: From design to application. J. Control. Release 332, 40-63, https://doi.org/10.1016/j.jconrel.2021.02.012.

George, A., Shah, P.A., Shrivastav, P.S., 2019. Natural biodegradable polymers based nano-formulations for drug delivery: A review. Int. J. Pharm. 561, 244-264. https:// doi.org/10.1016/j.ijpharm.2019.03.011.

Green, R.L., Thurau, G., Pixley, N.C., Mateos, A., Reed, R.A., Higgins, J.P., 2005. In-line monitoring of moisture content in fluid bed dryers using near-IR spectroscopy with consideration of sampling effects on method accuracy. Anal. Chem. 77 (14), 4515-4522. https://doi.org/10.1021/ac050272q10.1021/ac050272q.s001.

Grenier, J., Duval, H., Barou, F., Lv, P., David, B., Letourneur, D., 2019. Mechanisms of pore formation in hydrogel scaffolds textured by freeze-drying. Acta Biomater. 94, 195-203. https://doi.org/10.1016/j.actbio.2019.05.070.

Gun'ko, V., Savina, I., Mikhalovsky, S., 2017. Properties of water bound in hydrogels. Gels 3 (4), 37. https://doi.org/10.3390/gels3040037.

Holler, M., Raabe, J., Diaz, A., Guizar-Sicairos, M., Wepf, R., Odstrcil, M., Shaik, F.R., Panneels, V., Menzel, A., Sarafimov, B., Maag, S., Wang, X., Thominet, V., Walther, H., Lachat, T., Vitins, M., Bunk, O., 2018. OMNY - A tOMography Nano crYo stage. Rev. Sci. Instrum. 89 (4), 043706. https://doi.org/10.1063/1.5020247.

Jin, H., Nishiyama, Y., Wada, M., Kuga, S., 2004. Nanofibrillar cellulose aerogels. Colloids Surf., A 240 (1-3), 63-67. https://doi.org/10.1016/j.colsurfa.2004.03.007.

Kamat, M.S., Lodder, R.A., DeLuca, P.P., 1989. Near-infrared spectroscopic determination of residual moisture in lyophilized sucrose through intact glass vials. Pharm. Res. 6 (11), 961-965.

Kauppinen, A., Toiviainen, M., Korhonen, O., Aaltonen, J., Jarvinen, K., Paaso, J., Juuti, M., Ketolainen, J., 2013. In-line multipoint near-infrared spectroscopy for moisture content quantification during freeze-drying. Anal. Chem. 85 (4), 2377-2384. https://doi.org/10.1021/ac303403p.

Koivunotko, E., Merivaara, A., Niemelä, A., Valkonen, S., Manninen, K., Mäkinen, H., Viljanen, M., Svedström, K., Diaz, A., Holler, M., Zini, J., Paasonen, L., Korhonen, O., Huotari, S., Koivuniemi, A., Yliperttula, M., 2021. Molecular insights on successful reconstitution of freeze-dried nanofibrillated cellulose hydrogel. ACS Appl. Bio Mater. 4 (9), 7157-7167. https://doi.org/10.1021/acsabm.1c0073910.1021/ acsabm.1c00739.s001.

Lin, T.P., Hsu, C.C., 2002. Determination of residual moisture in lyophilized protein pharmaceuticals using a rapid and non-invasive method: near infrared spectroscopy. PDA J. Pharm. Sci. Technol. 56 (4), 196-205.

Merivaara, A., Zini, J., Koivunotko, E., Valkonen, S., Korhonen, O., Fernandes, F.M., Yliperttula, M., 2021. Preservation of biomaterials and cells by freeze-drying: Change of paradigm. J. Control. Release 336, 480-498. https://doi.org/10.1016/j. jconrel.2021.06.042.

Nagy, G., Király, G., Veres, P., Lázár, I., Fábián, I., Bánfalvi, G., Juhász, I., Kalmár, J., 2019. Controlled release of methotrexate from functionalized silica-gelatin aerogel microparticles applied against tumor cell growth. Int. J. Pharm. 558, 396-403. https://doi.org/10.1016/j.ijpharm.2019.01.024.

Najberg, M., Haji Mansor, M., Taillé, T., Bouré, C., Molina-Peña, R., Boury, F., Cenis, J.L., Garcion, E., Alvarez-Lorenzo, C., 2020. Aerogel sponges of silk fibroin, hyaluronic 
acid and heparin for soft tissue engineering: Composition-properties relationship Carbohydr. Polym. 237, 116107. https://doi.org/10.1016/j.carbpol.2020.116107.

Oddone, I., Barresi, A.A., Pisano, R., 2017. Influence of controlled ice nucleation on the freeze-drying of pharmaceutical products: the secondary drying step. Int. J. Pharm. 524 (1-2), 134-140. https://doi.org/10.1016/j.ijpharm.2017.03.077.

Parisi, C., Qin, K., Fernandes, F.M., 2021. Colonization versus encapsulation in cell-laden materials design: porosity and process biocompatibility determine cellularization pathways. Philos. Trans. Roy. Soc. A 379, 20200344. https://doi.org/10.1098/ rsta.2020.0344.

Paukkonen, H., Kunnari, M., Laurén, P., Hakkarainen, T., Auvinen, V.-V., Oksanen, T., Koivuniemi, R., Yliperttula, M., Laaksonen, T., 2017. Nanofibrillar cellulose hydrogels and reconstructed hydrogels as matrices for controlled drug release. Int. J. Pharm. 532 (1), 269-280. https://doi.org/10.1016/j.ijpharm.2017.09.002.

Pikal, M., Shah, S., Roy, M., Putman, R., 1990. The secondary drying stage of freeze drying: drying kinetics as a function of temperature and chamber pressure. Int. J. Pharm. 60 (3), 203-207.

Preskar, M., Korasa, K., Vrbanec, T., Klement, D., Vrečer, F., Gašperlin, M., 2021. Applicability of Raman and near-infrared spectroscopy in the monitoring of freezedrying injectable ibuprofen. Drug Dev. Ind. Pharm. 47 (5), 758-769. https://doi org/10.1080/03639045.2021.1934864.
Qin, K., Parisi, C., Fernandes, F.M., 2021. Recent advances in ice templating: from biomimetic composites to cell culture scaffolds and tissue engineering. J. Mater. Chem. B 9 (4), 889-907.

Rosas, J.G., de Waard, H., de Beer, T., Vervaet, C., Remon, J.P., Hinrichs, W.L.J., Frijlink, H.W., Blanco, M., 2014. NIR spectroscopy for the in-line monitoring of a multicomponent formulation during the entire freeze-drying process. J. Pharm. Biomed. Anal. 97, 39-46. https://doi.org/10.1016/j.jpba.2014.04.010.

Veres, P., López-Periago, A.M., Lázár, I., Saurina, J., Domingo, C., 2015. Hybrid aerogel preparations as drug delivery matrices for low water-solubility drugs. Int. J. Pharm. 496 (2), 360-370. https://doi.org/10.1016/j.ijpharm.2015.10.045.

Wakonig, K., Stadler, H.-C., Odstrčil, M., Tsai, E.H.R., Diaz, A., Holler, M., Usov, I., Raabe, J., Menzel, A., Guizar-Sicairos, M., 2020. PtychoShelves, a versatile highlevel framework for high-performance analysis of ptychographic data. J. Appl. Crystallogr. 53 (2), 574-586. https://doi.org/10.1107/S1600576720001776.

Weyer, L., Lo, S., 2006. Spectra-Structure Correlations in the Near-Infrared. In:

Chalmers, J.M., Griffiths, P.R. (Eds.), Handbook of Vibrational Spectroscopy. John Wiley \& Sons, Ltd, pp. 1817-1837.

Zaman, A., Huang, F., Jiang, M., Wei, W., Zhou, Z., 2020. Preparation, properties, and applications of natural cellulosic aerogels: A review. Energy Built Environ. 1 (1), 60-76. https://doi.org/10.1016/j.enbenv.2019.09.002. 